\title{
Communication Challenges Faced by Spanish-Speaking Caregivers of Children with Medical Complexity: a Qualitative Study
}

\author{
Savithri Nageswaran ${ }^{1}\left[\right.$ D Margaret B. Ellis $^{2} \cdot$ Mark S. Beveridge $^{3}$
}

Received: 28 June 2021 / Revised: 17 September 2021 / Accepted: 23 September 2021 / Published online: 30 September 2021

○ W. Montague Cobb-NMA Health Institute 2021

\begin{abstract}
Background Communication between caregivers and healthcare providers is important in the delivery of high-quality healthcare for children with medical complexity (CMC). Hispanic children face many challenges in access to healthcare services. Our objective was to describe the communication challenges faced by Spanish-speaking parents with limited English proficiency (SSP-LEP).

Methods This was a qualitative study of 70 children of Spanish-speaking caregivers, enrolled in a complex care program of a tertiary care children's hospital in North Carolina. Secondary source data were abstracted logs of care coordination tasks maintained by the program's two bilingual care coordinators for a median observation period of 45 months, and complemented by data from care coordinator interviews. Data were entered and coded in ATLAS.ti. Using thematic content analysis and an iterative process, we identified recurrent themes related to communication challenges of Spanish-speaking caregivers. Results Median age of children was 5 years; $51 \%$ were girls; 97\% had Medicaid; and 3\% were uninsured. Seven children died during the observation period. Three major themes were identified as follows: (1) caregivers faced many communication challenges primarily because of language barrier. (2) Multiple factors at caregiver, provider, and system levels, in addition to language barrier, contributed to communication challenges. (3) Communication challenges had serious consequences for CMC. These consequences were lessened by bilingual coordinators.

Conclusion SSP-LEP face unique communication challenges resulting in negative impact on the healthcare of their CMC. Bilingual coordinators can help improve communication between SSP-LEP and their healthcare providers. Interventions to address communication challenges of Spanish-speaking caregivers are warranted.
\end{abstract}

Keywords Children $\cdot$ Hispanic $\cdot$ Communication $\cdot$ Health services $\cdot$ Disparities

\begin{abstract}
Abbreviations
CMC Children with medical complexity

EMR Electronic Medical Record

SSP-LP Spanish-speaking parents/caregivers with limited English proficiency

LEP Limited English proficiency
\end{abstract}

Margaret B. Ellis and Mark S. Beveridge affiliations changed from Wake Forest School of Medicine, Winston-Salem, NC, USA.

Savithri Nageswaran

snageswa@wakehealth.edu

1 Department of Pediatrics, Wake Forest School of Medicine, 1 Medical Center Blvd, Winston-Salem, NC 27157, USA

2 Levine Children's Hospital, Atrium Health, Charlotte, NC, USA

3 Department of Pediatrics, UT Southwestern Medical Center, Dallas, TX, USA
WFHS Wake Forest Health Sciences

BCH Brenner Children's Hospital

CSHCN Children with special healthcare needs

PECP Pediatric Enhanced Care Program

\section{Background}

Children with medical complexity (CMC), a subgroup of children with special healthcare needs (CSHCN), have congenital or acquired health conditions that involve multiple organ systems [1,2]. CMC are medically fragile, may depend on technology for survival, and need many healthcare services [1]. Due to advances in medical care and technology, CMC are living longer [3]. While CMC constitute $<1 \%$ of all children, they account for $33 \%$ of healthcare expenditures for children [4]. CMC face many challenges 
with access to healthcare and are at increased risk of unmet healthcare needs [2].

Parents of CMC report that the healthcare system is fragmented and difficult to navigate $[5,6]$. Within the CSHCN population (of which CMC is a subgroup), difficulty using services, obtaining referrals to specialty care, and dissatisfaction with care are more prevalent in Hispanic children compared to non-Hispanic children [7, 8]. Although caregivers' ability to communicate with a child's health care team is vitally important, Spanish-speaking parents/caregivers are less satisfied with healthcare provider communication about their children than parents proficient in English [9, 10]. Children whose parents have limited English proficiency (LEP) are less likely to receive high-quality healthcare [11-14]. The association of parental LEP and resulting poor healthcare quality is particularly problematic for CSHCN $[15,16]$.

There is paucity of research on the healthcare experiences of CMC whose parents are Spanish-speaking and have LEP. Because CMC interface with the healthcare system more often than their counterparts do, the consequences of communication challenges due to parental LEP are likely to be amplified when compared to other children. Caring for CMC involves navigating a complex health system [5]; as such, Spanish-speaking parents with LEP (SSP-LEP) may face greater hardships advocating for their CMC. Therefore, the objectives of this paper are to describe the types of communication challenges faced by SSP-LEP, factors associated with these challenges, and their consequences on the healthcare received by CMC. Qualitative methodology is an ideal approach to glean insights into this complex and poorly understood topic. For purposes of this manuscript, we define communication as any verbal or written interaction between parents/caregivers (henceforth referred to as caregivers) and healthcare providers about healthcare or services for CMC.

\section{Methods}

This paper is part of a larger qualitative study about care coordination needs of CMC whose caregivers' primary language is Spanish. The methodology was described previously [17]. We report this paper according to the Standards for Reporting Qualitative Research guidelines (Appendix A) [18].

\section{Qualitative Approach and Research Paradigm}

The research paradigm for this study was interpretivist as our aim was to understand the real-life experiences of caregivers navigating the healthcare system for their children [19]. Encounter logs collected as part of clinical care served as a rich secondary source of data to answer our research questions that would not have been possible with other data sources. The approach was inductive analysis as we sought to identify concepts and ideas from the data $[19,20]$.

\section{Context}

The study was conducted at Brenner Children's Hospital $(\mathrm{BCH})$, a tertiary care children's hospital within the Wake Forest Health Sciences (WFHS) system located in WinstonSalem, NC. BCH serves a 19-county region with approximately 4,500 pediatric hospitalizations annually. Children included in the study were enrolled in the Pediatric Enhanced Care Program (PECP), a complex/palliative care program at $\mathrm{BCH}$. PECP consists of an interdisciplinary team of physicians, nurses, a social worker, and a patient navigator. The social worker and patient navigator (henceforth referred to as coordinators) are bilingual in English and Spanish. Children are eligible for PECP if they have a chronic condition that lasted/expected to last $\geq 12$ months, need care from $\geq 5$ specialists/services, or use $\geq 2$ types of technology (e.g., gastrostomy tube, tracheostomy tube). Demographic information of the 1,112 children enrolled in PECP at the time of the study are as follows: race/ethnicity $(n=960): 55 \%$ White, $21 \%$ Black, $18 \%$ Hispanic, $1 \%$ Asian, and 5\% other; health insurance $(n=1,066): 76 \%$ Medicaid, $8 \%$ both Medicaid and private, $15 \%$ private, and $1 \%$ uninsured.

The WFHS Institutional Review Board approved the study protocol. This is a retrospective cohort study. Children were included in this study if: (1) they were enrolled in PECP between December 6, 2011 and December 31, 2014, (2) their caregiver's primary spoken language was Spanish as indicated at program enrollment, and (3) they received services from one of the 2 bilingual coordinators of PECP. During the study period, 533 children were enrolled in PECP. Of these, 70 (13\%) children met the inclusion criteria and were included in the study cohort.

\section{Quantitative Data}

Child characteristics were obtained from information in a clinical REDCap database [21] that was gathered by the coordinators at each child's enrollment in PECP. Diagnoses were categorized into diagnostic groups based on underlying etiology. US Census data were used to determine counties' population density. [22] Counties were categorized into rural, suburban, and urban based on the population density of $\leq 250,251-750$, and $>750$ people per square mile, respectively. [23] Follow-up period was the number of days between the date of enrollment and February 28, 2015, or the date of death for children who died. Summary statistics were calculated in Microsoft Excel. 


\section{Qualitative Data}

Both coordinators provided longitudinal care for children through regular interactions with their caregivers. These interactions occurred in-person when children were hospitalized or at clinic visits, or by phone when they were at home. These contacts occurred periodically, but not at a set frequency. Coordinators contacted other healthcare providers to coordinate the care of children. Caregivers contacted coordinators by phone when they needed help. As interactions with caregivers and other healthcare providers occurred, coordinators entered the date of the encounter with a brief description about each encounter in an electronic database (referred to the "encounter log") for each child. Because the care coordinators' efforts were supported by 3 different funding sources, for maintaining their encounter logs, one used a database on REDCap, and the other a database that was part of a multi-centered care coordination collaborative. The encounter logs were not part of the child's electronic medical record (EMR).

Textual data in the encounter log, that contained care coordination tasks for each child from the time of enrollment until February 2015 or child's date of death, were extracted. Between January and April 2015, one of the authors (MSB) reviewed the data abstracted from the encounter log for each child with the coordinator involved in the child's care, one child at a time, in a series of in-person interviews. Forty children's logs were reviewed with one coordinator; 10 with the other; and 20 with both. During these reviews, existing information in the encounter logs for each child was clarified and additional information about the care coordination needs of the child was solicited. Written notes about each child obtained from coordinators during these reviews were added to the abstracted encounter log data. This review with coordinators enabled us to enrich the data from the encounter logs with information obtained directly from coordinators based on their recall. Thus, care coordination needs of the child that might not have been captured in the encounter logs were identified. We did not use an interview guide or audio-record interviews with care coordinators.

Qualitative data were entered into ATLAS.ti software, a tool designed specifically for qualitative data management. [24] Each child's encounter log, augmented by the text from coordinator review, was de-identified and entered as a separate document into ATLAS.ti. Data obtained from the encounter logs and from the coordinators were delineated within each document.

Two authors (SN and MSB) reviewed the qualitative data independently. A codebook was developed based on this review and revised as coding progressed (Appendix B). One author (MSB) coded the data using the ATLAS.ti software, which was then reviewed by another author (SN). Then, the authors compared and discussed each coded segment of the documents line by line until agreement on the coding scheme was reached. The final coding scheme (Appendix B) was applied to all documents.

Qualitative data were analyzed using thematic content analysis that provides a "map of the content and topics" across the dataset [20] and hence, is the appropriate analytical approach for our study aims. The content of each code was summarized by one author (MSB or $\mathrm{SN}$ ), and reviewed and validated by the other. By reviewing codes through an iterative process, recurrent themes and sub-themes were derived by their prevalence and salience in the data. Both authors discussed themes until agreement was reached. Analysis for this manuscript was limited to themes and subthemes about communication.

\section{Results}

Characteristics of children are presented in Table 1. Seventy CMC were observed for a median period of 535 days (range: 24 to 1,158 days). Seven children (10\%) died during the observation period. Demographic characteristics of CMC were - sex: 34 boys (49\%) and 36 girls (51\%); median age 5 years (range: 6 months to 18 years); insurance: 68 (97\%) had Medicaid and 2 (3\%) were uninsured; and residence: 56 (80\%) urban, 9 (13\%) rural, and $5(7 \%)$ suburban. Health conditions were as follows: chromosomal abnormality 15 (21\%); other genetic conditions 23 (33\%); neuromuscular

Table 1 Characteristics of children with medical complexity $(N=70)$

\begin{tabular}{ll}
\hline Characteristics & $n(\%)$ \\
\hline Sex & \\
Male & $34(49)$ \\
Female & $36(51)$ \\
Age, years (median, range) & $5(6$ months-18) \\
Residence & \\
Rural & $9(13)$ \\
Suburban & $5(7)$ \\
Urban & $56(80)$ \\
Health insurance & \\
Uninsured & $2(3)$ \\
Medicaid & $68(97)$ \\
Heath conditions & \\
Chromosomal abnormality & $15(21)$ \\
Other genetic conditions & $23(33)$ \\
Neuromuscular disorders & $8(12)$ \\
Prematurity & $5(7)$ \\
Anoxic brain injury & $11(16)$ \\
Other & $8(11)$ \\
Observation period, days (median, range) & $535(24-1,158)$ \\
Died during the study period & $7(10)$ \\
\hline
\end{tabular}


disorders 8 (12\%); prematurity 5 (7\%); anoxic brain injury $11(16 \%)$; and others $8(11 \%)$. Major themes identified that are related to communication are presented below. Illustrative quotes/notes are in italics. This is followed by the source of the quote/note - coordinator interview or encounter logs identified as "Care Coordinator Quote" or "Encounter Log," respectively. Numbers represent a child's unique identification number.

\section{Theme 1: Spanish-Speaking Caregivers with LEP Faced Many Types of Communication Challenges}

Most caregivers faced challenges in communication with their healthcare providers. Language barrier was an important contributor of challenges to in-person, telephone, and written communications.

\section{In-person Communication}

In the hospital and clinic settings, in-person or telephone interpreters were used by healthcare providers, but this did not occur consistently. Interpreters were not readily available in community agencies (e.g., pharmacy, home health nursing agency, equipment company). Without interpreters, caregivers communicated by resorting to gestures, or soliciting the help of English-speaking family members who would act as interpreters.

Patient's parents don't speak much English, so at first they communicated with their providers mostly through hand gestures. (Coordinator Quote about C08)

\section{Telephone Communication}

Phone calls to and from healthcare providers or agency representatives were frequent sources of miscommunication for families. Phone messages were most often left for families in English. Caregivers did not understand the messages and were not able to call back for clarification. Interpreters were used infrequently for phone interactions and, as such, caregivers were often confused about the content of the phone calls. Caregivers also experienced difficulty when making phone calls to providers because those who were answering the phone were English speakers. Additionally, caregivers had difficulty navigating the phone instructions and prompts in English. Caregivers did not feel empowered to request an interpreter if one was not offered. In some cases, even if caregivers requested interpreter help for phone communication, this service was not available or provided.

Language played a role in the pharmacy issue; mother cannot get interpreters to call her back to refill a prescription (Coordinator Quote about C06)
Mother receives a call from Equipment Company in English, confused about the meaning (Encounter Log for C17)

\section{Written Communication}

Language barrier was also a significant problem in written communications. Medication instructions were sometimes provided exclusively in English. Caregivers received appointment reminders or other documents in English and were not always able to decipher them. Other times, results of tests and procedures were sent to families in English. Caregivers could not comprehend letters and paperwork about benefits and resources including Social Security, Medicaid forms or expiration letters, application for camps, and Medicaid waiver program applications.

Mother receives letter in English from Social Security, tries calling to get an interpreter, but cannot do so successfully (Encounter Log for C01)

\section{Theme 2: Multiple Factors at Caregiver, Provider, and System Levels, in Addition to Language Barrier, Contributed to Communication Challenges}

\section{Caregiver-level Factors}

Practical factors contributed to communication challenges. Many caregivers used go-phones or pay-by-the-minute numbers; therefore, their phone numbers changed frequently. Oftentimes, a single phone was shared between multiple family members. In some cases, the phone number listed in the child's EMR was not accurate. This resulted in healthcare providers and agency representatives not being able to contact caregivers reliably. In addition, some phones did not have voicemail option available. Sometimes families did not return voice messages left on their phones.

Patient's father works at a distance from family, has the only cell phone, makes the family difficult to contact (Encounter Log for C03)

Equipment companies have a difficult time getting in touch with mom because of lack of reliable phone number (Coordinator Quote about C11)

Other caregiver-level factors that affected communication were caregivers' poor health literacy, lack of trust of healthcare providers, and inability to navigate the healthcare system. Families with poor health literacy were confused by instructions and did not follow them as a result. This resulted in children not receiving medications according to instructions on the prescription. 
Mother often misses appointments because she is not literate, cannot read the reminders (Encounter Log for C20)

Family is very shy, and won't ask questions of doctors directly; they ask questions only through the coordinator (Coordinator Quote about C24)

\section{System-level Factors}

Some caregivers did not understand the prescription refill system; they thought that they had to have another prescription instead of going directly to the pharmacy for refills. As a result, refills were delayed or missed. For controlled substances when identification was required by pharmacy, caregivers felt as though they were being discriminated against and did not understand that the system required identification for obtaining a controlled substance. Caregivers did not understand healthcare rules and regulations such as priorauthorization requirement for medications or procedures.

Mother thought she was being discriminated against because she was made to show passport to get medication (Coordinator Quote about C17)

\section{Provider-level Factors}

Several provider-level factors that contributed to communication problems were also identified. Pharmacies did not use interpreters either for their phone calls or when labeling their medications after purchase. When families attempted to call the pharmacies for refills, they were unable to communicate, and therefore, the refills were delayed. Surgeries and procedures were not explained resulting in misunderstanding about or delay in procedures.

Mom calling because she never received a call about test results (Encounter Log for C61)

Pharmacy gives 30-day supply instead of 90-day supply, pharmacy's mistake; once new prescription written mother was not notified (Encounter Log for C02)

\section{Theme 3: Communication Challenges Had Serious Consequences for CMC. Bilingual Coordinators Worked with Caregivers to Lessen These Consequences}

\section{Missed Appointments}

The consequences of communication issues varied but affected both CMC and their caregivers. A common problem identified was missed appointments. When appointment reminders were sent or voice messages left in English, children missed these appointments. When there were appointment conflicts, caregivers were unable to call providers' offices to reschedule these appointments.

Patient missed appointment because they never received a call from [specialty] clinic (Encounter Log for $\mathrm{C} 01$ )

Mom missed appointment because she did not know the time (Encounter Log for C04)

\section{Medication Issues}

Medication issues were another common consequence of miscommunication or misunderstanding. CMC experienced delayed or missed refills because of caregivers' lack of understanding of the system or inability of the caregiver to communicate with the pharmacy. Incorrect administration of medication and failure to administer medication were also seen.

Patient has gone for months without supplies because mother didn't know who to call (Coordinator Quote about C17)

Patient took medication incorrectly because family spoke with a non-Spanish speaking nurse, causing a misunderstanding about how it is taken and when (Coordinator Quote about C19)

\section{Missed or Delayed Services}

Communication challenges resulted in missed or delayed services for some children. Children did not receive health and support services they were qualified for, such as early intervention services or special camps for CMC. One child had a surgical procedure delayed for months due to a miscommunication between the surgical team and the caregiver. Due to misconceptions about helpfulness of the procedure, one family missed multiple appointments delaying a diagnosis of seizure and appropriate seizure control for their daughter. Caregivers' inability to complete paperwork needed to obtain services resulted in missed or delayed services for children.

Mom was not responding to letters written in English asking her for supplies she needed, and consequently she stopped receiving supplies (Encounter Log for C06)

\section{Caregiver Consequence}

Caregivers were unable to advocate for services for their children secondary to the language barrier. They experienced frustration as a result of communication challenges surrounding the care of their children. Communication 
problems contributed to lack of caregiver trust of providers that resulted in less adherence to medical instructions.

Mom concerned about patient, but cannot contact PCP because of language barrier, since she cannot negotiate Spanish line (Encounter Log for C29)

Family was buying formula that should have been paid for because they could not negotiate the process. (Encounter Log for C38)

Mom is upset at home health because G-tube fell out at home; they are unable to say exactly when. (Encounter Log for C53)

Poor communication in the past from providers (e.g. wanting to remove tonsils along with ear tubes, didn't tell mother why) has made mom very distrustful of providers. (Care Coordinator Quote about C12)

\section{Care Coordinators}

Bilingual care coordinators identified caregivers' communication challenges and performed many roles to address these challenges (Table 2). They served as intermediaries in communication between caregivers and healthcare providers in the hospital and in the community. They also assisted caregivers navigate the healthcare system and educated them about the process (e.g., how to refill medications). Some caregivers who had difficulty understanding instructions/ messages provided in English reached out to the coordinators for assistance interpreting these messages.

Care coordinator assisted mom in reading 2 letters, one from Social Security and the other from Medicaid transportation (Encounter Log for C26)

Coordinators helped caregivers by checking appointment time and location, scheduling/rescheduling appointments, providing directions to the appointment location, arranging for transportation for appointments, and negotiating with providers about appointments.

Mother calls to verify time and place of appointment (Encounter Log for C13)

\section{Discussion}

We found that SSP-LEP of CMC faced challenges with in-person, telephone, and written communications due to language barrier. In addition to language barrier, our study showed that other factors at the caregiver, provider, and system levels contributed to the communication challenges experienced by SSP-LEP. Poor communication had

Table 2 Care coordinators' encounters to address communication challenges of Spanish-speaking caregivers of children with medical complexity

1. Serve as interpreters in helping caregivers understand communication from healthcare providers (e.g., clinic, hospital), representatives of community agencies (e.g., pharmacy, equipment supply companies, home health agency), and administrative agencies

a. Help interpret phone calls that caregivers received

b. Help caregivers make phone calls to providers/agencies

c. Interpret letters, forms, and documents that are sent to the child

d. Interpret in-person communication with providers in the hospital and clinic

2. Help navigate the healthcare system by performing the tasks typically carried out by caregivers of children with medical complexity

a. Call clinics to schedule/change appointments*

b. Call pharmacy to refill medications*

c. Call equipment supply companies to order supplies

d. Complete application forms for camps and special services (e.g., home and community-based waiver applications)

e. Complete required processes to obtain services (e.g., transportation)

3. Educate and guide caregivers about ways to navigate the healthcare system

a. Provide education about the process to refill prescription medications

b. Provide guidance about obtaining non-emergency medical transportation $\dagger$

c. Empower caregivers to advocate for health services for their children

4. Identify resources in the community to meet the practical needs of the child and family $†$

5. Serve as a liaison between caregiver and healthcare providers

a. Clarify with caregiver, rationale for services (e.g., school, early intervention services) tests and procedures, and clarify appointment time/location

b. Provide clarification to caregivers about management plan and recommendations of healthcare providers

c. Communicating with healthcare providers about caregivers' wishes and goals for their children

6. Provide emotional support to caregivers, and advocate with healthcare providers and agencies about health and support services for the child (e.g., writing letters for immigration agency)

*Most frequently reported in care coordinator encounter logs

${ }^{\dagger}$ Reported previously in other manuscripts from this project 
consequences for $\mathrm{CMC}$ including missed clinic appointments, delay in getting needed services, and medication non-adherence.

Many previous reports have shown language barrier to be an important factor in children of SSP-LEP experiencing poor communication and not receiving needed healthcare services as a result. $[12,13,16,25,26]$ Research also shows that healthcare communication challenges are often mitigated by the use of medical interpreters [14, 26, 27]. We found, however, that caregivers had difficulty accessing interpreters in medical settings outside the hospital, such as pharmacies, and other community sites. In a prior survey of pharmacists, only $55 \%$ were satisfied with their communication with patients with LEP; a large majority did not have access to interpreters. [28] Studies have shown that interpreters were more likely to be available in hospitals or hospital-based clinics; even in these settings, visits for Spanish-speaking families were frequently conducted in English. [9, 26] Machine translation and interpretation services are increasingly available and have the potential to address the challenges of language barrier in healthcare communications. [29] Healthcare providers should be aware of the communication challenges faced by SSP-LEP, advocate for resources to address these challenges (e.g., interpreter services available outside the hospital setting), and make an effort to improve communication with SSP-LEP in clinical practice.

We found multiple factors contributing to communication challenges faced by SSP-LEP of CMC, in addition to language barrier. Previous reports highlight the importance of illiteracy, poor health literacy, [30, 31] and cultural factors contributing to healthcare communication challenges for this population. [32, 33]s In our study, system-level factors — providers sending forms, letters, and instructions, and leaving phone messages in English - made it difficult for SSP-LEP to access services for their CMC. This is because the healthcare system is designed for English-speaking patients and can be exceedingly difficult to navigate for those who prefer to speak Spanish. For example, during the recent coronavirus pandemic, when telehealth services were expanded, we observed that the process to access telehealth in our institution was available only in English; this made it difficult for CMC of SSP-LEP to receive telehealth services. Healthcare institutions and programs should consider the needs of SSP-LEP when structuring and making changes to systems and processes.

Poor communication had consequences for CMC including missed clinic appointments, delay in getting needed services, and medication non-adherence. Our findings are consistent with prior research that showed delays in diagnosis and treatment of children whose parents had LEP. [11-14] Prior studies have reported the difficulties faced by SSP-LEP in navigating the health system for their children.
$[32,34]$ Bilingual care coordinators could play a key role in mitigating the consequences of parental communication challenges for CMC. Flores et al., in a randomized controlled trial, showed that bilingual care coordinators with specialized training facilitated SSP-LEP to obtain health insurance for their children. [35] National organizations have recommended that $\mathrm{CMC}$ have access to an interdisciplinary care team that can address the unique needs of CMC and their families. [36] However, care coordinators' services, as described in our study, are not always reimbursed by health insurance plans. Changes at the policy level are needed to have care coordination services accessible to all CMC. Inclusion of coordinators, especially those with expertise in helping SSP-LEP in complex care teams, could be a strategy to address the unique needs of these children.

Our study has certain limitations. Because of the qualitative nature of this study, we were unable to quantify parental LEP and the extent of the communication problems faced by SSP-LEP. Since this study is regional in scope, the experiences of SSP-LEP may not be representative of all caregivers of CMC in the USA. Although the data from encounter logs was augmented with data from interviews, qualitative data may not have captured all care coordination activities. Since we used secondary source data that were already collected, we could not perform validity checks of the encounter logs. We relied on care coordinators' logs and perceptions to understand caregiver experiences, and did not obtain information directly from caregivers. This could have resulted in recall bias. Also, our study findings may or may not reflect caregiver perspective on communication barriers. Caregiver perspectives on communication will be valuable to identify communication challenges as well as potential solutions. Finally, we did not systematically assess English language proficiency of caregivers at the time of enrollment in PECP. Future studies should evaluate whether communication challenges identified in our study vary by the extent of parents' LEP.

\section{Conclusion}

SSP-LEP of CMC face many communication challenges that negatively affect access to and quality of health services for these children. The role of bilingual care coordinators in addressing these challenges requires further evaluation.

Supplementary Information The online version contains supplementary material available at https://doi.org/10.1007/s40615-021-01161-x.

Acknowledgements We thank Ms. Aura Rosado and Ms. Vanessa Ortiz for participating in interviews about children with medical complexity, and Dr. Emily Ware MD, MSN for assistance with literature review. 
Author Contribution All authors have read and approved the manuscript.

SN conceptualized and designed the study, analyzed the data, interpreted the results, drafted the manuscript, approved the submitted version, and agreed to be personally accountable for the authors' contributions and the accuracy or integrity of any part of the work.

MBE interpreted the results, conducted literature review, revised the manuscript, approved the submitted version, and agreed to be personally accountable for the authors' contributions and the accuracy or integrity of any part of the work.

MSB collected data, analyzed data, interpreted the results, revised the manuscript, approved the submitted version, and agreed to be personally accountable for the authors' contributions and the accuracy or integrity of any part of the work.

Funding Dr. Nageswaran was supported by Healthy Tomorrow's Partnership for Children Program Grant from the Health Resources and Service Administration (H17MC11228) and Integrated Community Systems for CSHCN Grant from the Health Resources and Service Administration (D70MC23061).

Data Availability The qualitative dataset generated and/or analyzed during the current study are not publicly available because of the potential for compromising privacy of individuals, but summarized data are available from the corresponding author on reasonable request.

Code Availability Not applicable.

\section{Declarations}

Ethics Approval and Consent to Participate The Wake Forest Health Sciences Institutional Review Board approved the study protocol. Written informed consent was waived. This manuscript reports aggregate data and does not report private information from any one individual.

Conflict of Interest The authors declare no competing interests.

\section{References}

1. Cohen E, Kuo DZ, Agrawal R, et al. Children with medical complexity: an emerging population for clinical and research initiatives. Pediatrics. 2011;127(3):529-38.

2. Kuo DZ, Goudie A, Cohen E, et al. Inequities in health care needs for children with medical complexity. Health Aff (Millwood). 2014;33(12):2190-8.

3. Feudtner C, Christakis DA, Zimmerman FJ, Muldoon JH, Neff JM, Koepsell TD. Characteristics of deaths occurring in children's hospitals: implications for supportive care services. Pediatrics. 2002;109(5):887-93.

4. Cohen E, Berry JG, Camacho X, Anderson G, Wodchis W, Guttmann A. Patterns and costs of health care use of children with medical complexity. Pediatrics. 2012;130(6):e1463-1470.

5. Golden SL, Nageswaran S. Caregiver voices: coordinating care for children with complex chronic conditions. Clin Pediatr (Phila). 2012;51(8):723-9.

6. Kuo DZ, Cohen E, Agrawal R, Berry JG, Casey PH. A national profile of caregiver challenges among more medically complex children with special health care needs. Arch Pediatr Adolesc Med. 2011;165(11):1020-6.

7. Ngui EM, Flores G. Satisfaction with care and ease of using health care services among parents of children with special health care needs: the roles of race/ethnicity, insurance, language, and adequacy of family-centered care. Pediatrics. 2006;117(4):1184-96.

8. Ngui EM, Flores G. Unmet needs for specialty, dental, mental, and allied health care among children with special health care needs: are there racial/ethnic disparities? J Health Care Poor Underserved. 2007;18(4):931-49.

9. Flower KB, Skinner AC, Yin HS, et al. Satisfaction with communication in primary care for Spanish-speaking and Englishspeaking parents. Acad Pediatr. 2017;17(4):416-23.

10. Jaramillo J, Snyder E, Dunlap JL, Wright R, Mendoza F, Bruzoni M. The Hispanic Clinic for Pediatric Surgery: a model to improve parent-provider communication for Hispanic pediatric surgery patients. J Pediatr Surg. 2016;51(4):670-4.

11. Levas MN, Cowden JD, Dowd MD. Effects of the limited English proficiency of parents on hospital length of stay and home health care referral for their home health care-eligible children with infections. Arch Pediatr Adolesc Med. 2011;165(9):831-6.

12. Cohen AL, Rivara F, Marcuse EK, McPhillips H, Davis R. Are language barriers associated with serious medical events in hospitalized pediatric patients? Pediatrics. 2005;116(3):575-9.

13. Levas MN, Dayan PS, Mittal MK, et al. Effect of Hispanic ethnicity and language barriers on appendiceal perforation rates and imaging in children. J Pediatr. 2014;164(6):1286-1291 e1282.

14. Jimenez N, Jackson DL, Zhou C, Ayala NC, Ebel BE. Postoperative pain management in children, parental English proficiency, and access to interpretation. Hosp Pediatr. 2014;4(1):23-30.

15. Eneriz-Wiemer M, Sanders LM, Barr DA, Mendoza FS. Parental limited English proficiency and health outcomes for children with special health care needs: a systematic review. Acad Pediatr. 2014;14(2):128-36.

16. Yu SM, Singh GK. Household language use and health care access, unmet need, and family impact among CSHCN. Pediatrics. 2009;124(Suppl 4):S414-419.

17. Nageswaran S, Rosado AI, Beveridge MS Jr. Challenges faced by Latino caregivers in transportation of children with medical complexity. N C Med J. 2018;79(6):358-64.

18. O'Brien BC, Harris IB, Beckman TJ, Reed DA, Cook DA. Standards for reporting qualitative research: a synthesis of recommendations. Acad Med. 2014;89(9):1245-51.

19. Ravitch SM, Carl NM. Qualitative research: bridging the conceptual, theoretical, and methodological. 2nd ed. Los Angeles: Sage; 2021.

20. Green J, Thorogood N. Qualitative methods for health research.: Sage Publications; 2018.

21. Research Electronic Data Capture. Clinical \& translational science awards. http://project-redcap.org/.

22. U.S. Census Bureau: QuickFacts. https://www.census.gov/quick facts/. Accessed 7/12/2017.

23. The Rural Center: Rural county map. http://www.ncruralcenter. org/rural-county-ma. Accessed 7/12/2017.

24. ATLAS.ti. Qualitative data analysis [computer program]. Version 7.2. Berlin, Germany: Atlas.ti Scientific Software Development $\mathrm{GmbH} ; 2013$.

25. Highland KB, Lundahl A, Kidwell KM, Hankey M, Caballos M, McChargue D. Latina and non-Latina mothers' perceived health barriers and benefits and their relationship to children's health behaviors. Matern Child Health J. 2016;20(6):1305-13.

26. Gutman CK, Cousins L, Gritton J, et al. Professional interpreter use and discharge communication in the pediatric emergency department. Acad Pediatr. 2018;18(8):935-43.

27. Flores $\mathrm{G}$. The impact of medical interpreter services on the quality of health care: a systematic review. Medical care research and review : MCRR. 2005;62(3):255-99.

28. Bradshaw M, Tomany-Korman S, Flores G. Language barriers to prescriptions for patients with limited English proficiency: a survey of pharmacies. 2007;120(2):e225-e235 
29. Dew KN, Turner AM, Choi YK, Bosold A, Kirchhoff K. Development of machine translation technology for assisting health communication: a systematic review. J Biomed Inform. 2018;85:56-67.

30. Yu SM, Nyman RM, Kogan MD, Huang ZJ, Schwalberg RH. Parent's language of interview and access to care for children with special health care needs. Ambul Pediatr. 2004;4(2):181-7.

31. Leyva M, Sharif I, Ozuah PO. Health literacy among Spanishspeaking Latino parents with limited English proficiency. Ambul Pediatr. 2005;5(1):56-9.

32. Gannotti ME, Kaplan LC, Handweker WP, Groce NE. Cultural influences on health care use: differences in perceived unmet needs and expectations of providers by Latino and EuroAmerican parents of children with special health care needs. 2004;25(3):156-165

33. Munoz-Blanco S, Raisanen JC, Donohue PK, Boss RD. Enhancing pediatric palliative care for Latino children and their families: a review of the literature and recommendations for research and practice in the United States. Children-Basel. 2018;5(1).

34. Flores G, Abreu M, Brown V, Tomany-Korman SC. How Medicaid and the State Children's Health Insurance Program can do a better job of insuring uninsured children: the perspectives of parents of uninsured Latino children. Ambul Pediatr. 2005;5(6):332-40.

35. Flores G, Abreu M, Chaisson CE, et al. A randomized, controlled trial of the effectiveness of community-based case management in insuring uninsured Latino children. Pediatrics. 2005;116(6):1433-41.

36. Kuo DZ, McAllister JW, Rossignol L, Turchi RM, Stille CJ. Care coordination for children with medical complexity: whose care is it, anyway? Pediatrics. 2018;141(Suppl 3):S224-32.

Publisher's Note Springer Nature remains neutral with regard to jurisdictional claims in published maps and institutional affiliations. 